Spin-lattice coupling in uranium dioxide probed by magnetostriction measurements at high magnetic fields (P08358-E001-PF)

K. Gofryk, M. Jaime

December 2014

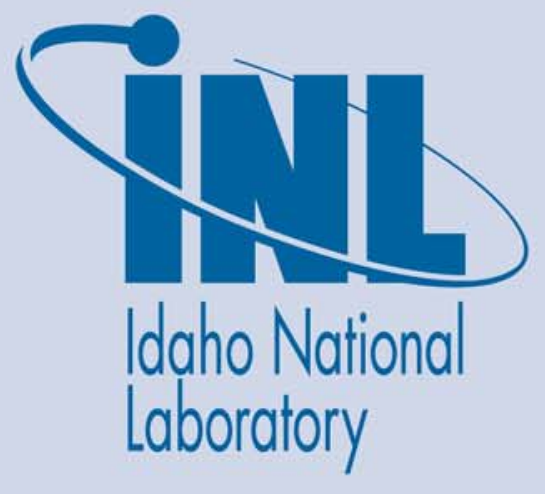

The INL is a U.S. Department of Energy National Laboratory operated by Battelle Energy Alliance 
INL/EXT-14-33975

\title{
Spin-lattice coupling in uranium dioxide probed by magnetostriction measurements at high magnetic fields (P08358-E001-PF)
}

\author{
K. Gofryk, M. Jaime
}

December 2014

\author{
Idaho National Laboratory \\ Idaho Falls, Idaho 83415
}

http://www.inl.gov

Prepared for the

U.S. Department of Energy

Office of Nuclear Energy

Under DOE Idaho Operations Office

Contract DE-AC07-05ID14517 


\title{
NATIONAL HIGH MAGNETIC FIELD LABORATORY 2014 ANNUAL RESEARCH REPORT
}

\section{Spin-lattice coupling in uranium dioxide probed by magnetostriction measurements at high magnetic fields (P08358-E001-PF)}

\author{
Gofryk, K. (Idaho National Laboratory); Jaime, M. (National High Magnetic Field Laboratory LANL)
}

\section{Introduction}

Uranium dioxide is known to be a Mott insulator and to develop a first order antiferromagnetic ordering of uranium magnetic moments below $30 \mathrm{~K}$. Strong spin-lattice interactions based on Jahn-Teller interactions have been proposed to account for these behaviors [1-3]. Despite huge theoretical and experimental efforts the nature of the strong spin-lattice coupling and how the coupling affects the thermal properties of $\mathrm{UO}_{2}$ are still unclear.

\section{Experimental}

The magnetostriction has been measured on oriented high quality single crystals of uranium dioxide using optical fiber Bragg grating (FBG) dilatometry. The measurements have been performed in pulsed magnetic fields up to $65 \mathrm{~T}$ (NHMFL LANL).

\section{Results and Discussion}

Figure 1a shows the temperature dependence of the thermal expansion $\Delta \mathrm{L} / \mathrm{L}$ and thermal expansion coefficient $\alpha$ of $\mathrm{UO}_{2}$ crystal measured along $<100>$ direction. A sharp first order like anomaly is observed at about $30 \mathrm{~K}$ associated with antiferromagnetic phase transition and simultaneous JahnTeller distortion. In Figure $1 \mathrm{~b}$ selected representative isothermal magnetostriction curves are presented for $<100\rangle$ and $<111>$ directions. As seen, in paramagnetic state the magnetostriction is negative and much stronger for $<111>$ axis. In the magnetic state a positive magnetostriction is observed together with irreversible behavior at low fields. This behavior has only been observed in zero-field-cooled conditions. At higher fields the magnetostriction forms a broad maximum. Solid arrows in Figure $1 \mathrm{~b}$ mark position of these

(a)

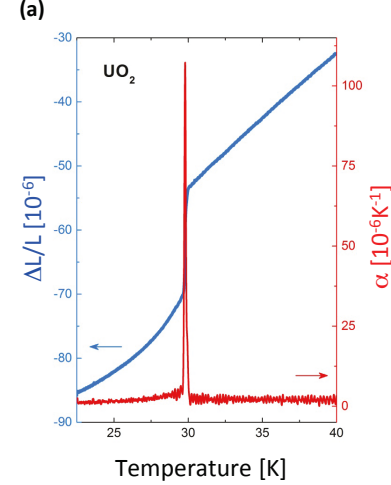

Temperature $[\mathrm{K}]$

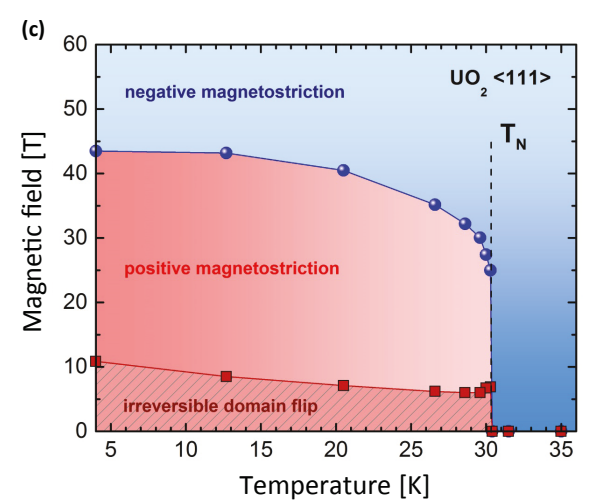

rigure 1. (a) Ine temperature dependence of the thermal expansion (left axis) and thermal expansion coefficient (right axis) of $\mathrm{UO}_{2}$ single crystal. (b) Isothermal magnetostriction measured above and below $\mathrm{T}_{\mathrm{N}}$. Solid arrows mark positions of anomalies used to construct the phase diagram. (c) The phase diagram of uranium dioxide obtained for <111> direction (see text). anomalies and by tracking the evolution of these features, a phase diagram can be constructed as presented in Figure 1c for $<111>$ direction. At the present time we are unable to construct a similar diagram for the $<100>$ direction. For several magnetostriction measurement attempts performed so far below the Neel temperature in this orientation the sample has detached off the optical fiber at about $45 \mathrm{~T}$.

\section{Conclusions}

Our preliminary magnetostriction measurements have already shown a strong interplay of lattice dynamic and magnetism in both antiferromagnetic and paramagnetic states, and give unambiguous evidence of strong spinphonon coupling in uranium dioxide. Further studies are planned to address the puzzling behavior of $\mathrm{UO}_{2}$ in magnetic and paramagnetic states and details of the spin-phonon coupling.

\section{Acknowledgements}

A portion of this work was performed at the National High Magnetic Field Laboratory, which is supported by National Science Foundation Cooperative Agreement No. DMR-1157490, the State of Florida, and the U.S. Department of Energy. Work at Idaho National Laboratory was partially supported by NEAMS program.

\section{References}

[1] Allen, S. J. Phys. Rev. 166, 530 (1968) and Allen, S. J. Phys. Rev. 167, 492 (1968).

[2] Caciuffo, R. et al., Phys. Rev. B 59, 13892 (1999) and Cowley, R. A. et al. Phys. Rev. Lett. 16, 683 (1966).

[3] K. Gofryk, K. et al. Nature Communications 5, 4551 (2014). 